\title{
Validação do Instrumento de Avaliação de Materiais Educativos Impressos com foco no Letramento em Saúde para o Brasil (AMEELS-BR)
}

Validation of the Instrument for the Evaluation of Printed Educational Materials with focus on Health Literacy for Brazil (AMEELS-BR)

Validación del Instrumento de Evaluación de Materiales Educativos Impresos con enfoque en Alfabetización en Salud para Brasil (AMEELS-BR)

Recebido: 01/09/2021 | Revisado: 08/09/2021 | Aceito: 10/09/2021 | Publicado: 13/09/2021

\author{
Raquel Bezerra de Abreu \\ ORCID: https://orcid.org/0000-0003-2152-0921 \\ Universidade Estadual do Ceará, Brasil \\ E-mail: raquel.b.abr@gmail.com \\ Antônio Augusto Ferreira Carioca \\ ORCID: https://orcid.org/0000-0002-1194-562X \\ Universidade de Fortaleza, Brasil \\ E-mail: nutriaugusto@gmail.com \\ Helena Alves de Carvalho Sampaio \\ ORCID: https://orcid.org/0000-0001-5353-8259 \\ Universidade Estadual do Ceará, Brasil \\ E-mail: dr.hard2@gmail.com \\ Cláudia Machado Coelho Souza de Vasconcelos \\ ORCID:https://orcid.org/0000-0002-3395-6143 \\ Universidade Estadual do Ceará, Brasil \\ E-mail: claudia.vasconcelos@uece.br
}

\begin{abstract}
Resumo
Objetivo: Validar o conteúdo do Instrumento de Avaliação de Materiais Educativos Impressos com foco no Letramento em Saúde para o Brasil (AMEELS-BR). Metodologia: Estudo metodológico descritivo, do tipo validação de conteúdo com a metodologia de Pasquali, realizado com 14 docentes da área da saúde e da pedagogia, que avaliaram os itens do instrumento por meio de classificação item a item pela escala Likert. A concordância entre os juízes foi verificada de forma quantitativa por meio do Índice de Validade de Conteúdo (IVC). Resultados: Dos 53 itens que compõem o instrumento, 39 itens obtiveram concordância dentro do nível estabelecido (IVC >0,78), enquanto 14 não obtiveram o nível de concordância necessário, com valores de IVC entre 0,54 e 0,77. Dessa forma, optou-se por reformular os itens que não atingiram o IVC necessário. O instrumento final ficou com 55 itens, pois 02 itens foram subdivididos, acatando recomendação dos juízes. Quanto à avaliação geral do instrumento, resultou em IVC $=0,81$, quando realizado por meio da soma de todos os IVC calculados separadamente dividido pelo número de itens considerado na avaliação. Portanto, o instrumento apresentou a concordância mínima para a verificação da validade de um novo instrumento, que não deve ser inferior a 0,8. Conclusão: O AMEELS-BR, de forma geral, foi considerado válido quanto ao seu conteúdo pelo painel de especialistas, apresentando índice de validade dentro dos parâmetros aceitáveis e podendo assim ser utilizado por gestores e profissionais de saúde para a avaliação e elaboração dos MEI.
\end{abstract}

Palavras-chave: Educação em saúde; Estudo de validação; Letramento em saúde.

\begin{abstract}
Objective: Validate the content of the Instrument for the Evaluation of Printed Educational Materials with focus on Health Literacy for Brazil (AMEELS-BR). Methodology: Descriptive methodological study, of the content validation type using Pasquali 's methodology, carried out with 14 professors from the health and pedagogy area, who evaluated the instrument's items through an item-by-item classification using the Likert scale. The agreement between the judges was verified quantitatively through the Content Validity Index (CVI). Results: Of the 53 items that make up the instrument, 39 items obtained agreement within the established level (CVI >0.78), while 14 did not obtain the necessary agreement level, with CVI values between 0.54 and 0.77 . Thus, it was decided to reformulate the items that did not reach the necessary CVI. The final instrument had 55 items, as 02 items were subdivided, following the judges' recommendation. As for the general assessment of the instrument, it resulted in CVI=0.81, when performed through the sum of all CVI calculated separately, divided by the number of items considered in the assessment. Therefore, the instrument presented the minimum agreement for verifying the validity of a new instrument, which
\end{abstract}


should not be less than 0.8. Conclusion: The AMEELS-BR, in general, was considered valid in terms of its content by the expert panel, presenting a validity index within acceptable parameters and thus being able to be used by managers and health professionals for the evaluation and elaboration of the MEI.

Keywords: Health education; Validation study; Health literacy.

\section{Resumen}

Objetivo: Validar el contenido del Instrumento de Evaluación de Material Educativo Impreso enfocado en Alfabetización en Salud para Brasil (AMEELS-BR). Metodología: Estudio metodológico descriptivo, tipo validación de contenido con la metodología Pasquali, realizado con 14 profesores de las áreas de salud y pedagogía, quienes evaluaron los ítems del instrumento mediante clasificación ítem por ítem mediante la escala Likert. La concordancia entre los jueces se verificó cuantitativamente a través del Índice de Validez de Contenido (IVC). Resultados: De los 53 ítems que componen el instrumento, 39 ítems obtuvieron concordancia dentro del nivel establecido (IVC>0,78), mientras que 14 no obtuvieron el nivel de concordancia necesario, con valores de IVC entre 0,54 y 0,77. Así, se decidió reformular los ítems que no alcanzaron el IVC necesario. El instrumento final contó con 55 ítems, ya que se subdividieron 02 ítems, siguiendo la recomendación de los jueces. En cuanto a la evaluación general del instrumento, resultó en IVC $=0,81$, cuando se realizó mediante la suma de todos los IVC calculados por separado, dividida por el número de ítems considerados en la evaluación. Por tanto, el instrumento presentó el acuerdo mínimo para verificar la validez de un nuevo instrumento, que no debe ser inferior a 0,8. Conclusión: El AMEELS-BR, en general, fue considerado válido en cuanto a su contenido por el panel de expertos, presentando un índice de validez dentro de parámetros aceptables y pudiendo así ser utilizado por gestores y profesionales de la salud para la evaluación y elaboración del MEI.

Palabras clave: Educación para la salud; Estudio de validación; Alfabetización en salud.

\section{Introdução}

Dentre estratégias amplamente utilizadas para melhorar a qualidade da prática dos profissionais de saúde e os resultados do paciente encontram-se os materiais educativos impressos (MEI) (Giguère et al., 2020). O uso dos MEI tem por objetivo informar e reforçar as orientações transmitidas oralmente pelos profissionais de saúde, bem como facilitar o autocuidado, visto que é também um recurso disponível para dúvidas que possam surgir quando eles estão distantes dos profissionais (Nascimento et al., 2015).

Entretanto, diversas vezes as informações se apresentam de forma complexa, com a utilização de linguagem formal ou terminologia médica, o que pode comprometer a compreensão, afetando o entendimento e a qualidade do cuidado, consequentemente (Santos et al., 2017). Assim sendo, o propósito educacional poderá ser limitado ou inexistente, sendo de extrema importância a avaliação desses materiais no que diz respeito à linguagem, conteúdo e aspectos gráficos como layout, design e ilustrações (Mialhe \& Costa, 2008) considerando também o grau de letramento em saúde dos indivíduos (Rocha \& Lemos, 2016).

Entende-se por letramento em saúde (LS) como o conhecimento, motivação e competências das pessoas para acessar, entender, avaliar e aplicar informação em saúde, fazendo julgamentos e tomando decisões no dia a dia no que tange ao cuidado da saúde, prevenção de doenças e promoção da saúde para manter ou melhorar a qualidade de vida (Sørensen et al., 2012).

Em outros países, as pesquisas de avaliação de materiais educativos impressos em saúde já constituem um campo explorado, mas no Brasil são encontrados poucos estudos (Hoffmann \& Worrall, 2004; Vasconcelos, Sampaio, \& Vergara, 2018). Paralelamente a pouca produção científica sobre a temática, são produzidos e disponibilizados pelo governo federal brasileiro uma gama de materiais educativos envolvendo várias temáticas (Vasconcelos, Sampaio, \& Vergara, 2018). No entanto, ainda são poucos os estudos que avaliam materiais educativos, principalmente considerando os princípios do LS (Passamai et al., 2012; Vasconcelos, Sampaio, \& Vergara, 2018).

Dentre os instrumentos utilizados para a avaliação de materiais educativos, destaca-se o Suitability Assessment of Materials (SAM) (Doak \& Doak, 1996), que já foi utilizado em pesquisas brasileiras e foi traduzido em língua portuguesa (Sousa, Turrini, \& Poveda, 2015). Assim, a literatura nesse campo, em maioria, cita instrumentos para avaliação de MEI em 
estudos estrangeiros, evidenciando a inexistência de uma ferramenta validada no Brasil que contemple os pressupostos do LS (Vasconcelos, Sampaio, \& Vergara,2018).

Dessa maneira, tendo em vista o potencial dos materiais educativos como estratégia para promoção de saúde e a necessidade de aprimorar políticas e ações já existentes no país, e a lacuna do conhecimento sobre avaliação de materiais educativos, se propôs o Instrumento de Avaliação de Materiais Educativos Impressos com foco no Letramento em Saúde para o Brasil (AMEELS-BR), desenvolvido a partir da tese de Vasconcelos (2016), intitulada "Materiais educativos para prevenção e controle de doenças crônicas: uma avaliação à luz dos pressupostos do Letramento em Saúde.”. O referido instrumento já foi avaliado quanto a sua reprodutibilidade (Nascimento et al., 2018) e agora realizou-se uma validação de conteúdo.

Considerando a relevância da elaboração e uso de materiais educativos e visto que não há no Brasil instrumento para avaliação de materiais educativos escritos com foco no letramento em saúde validado, esse estudo tem por objetivo validar o conteúdo do Instrumento de Avaliação de Materiais Educativos Impressos com foco no Letramento em Saúde para o Brasil (AMEELS-BR).

\section{Metodologia}

Estudo metodológico descritivo, do tipo validação de conteúdo com a metodologia de Pasquali (2010), realizado no período de agosto de 2019 a julho de 2020, com 14 docentes da área da saúde e da pedagogia para validação de instrumento para avaliação de materiais educativos impressos, previamente elaborado como parte de um projeto de pesquisa intitulado "Plano Alfa Saúde: aplicação dos pressupostos do letramento em saúde e da formação de navegadores na capacitação de equipes do SUS". A validação do instrumento foi apoiada no referencial metodológico proposto por Pereira et al. (2018) e Pasquali (2010).

O instrumento foi intitulado Instrumento de Avaliação de Materiais Educativos Impressos com foco no Letramento em Saúde para o Brasil (AMEELS-BR) e sua elaboração consistiu nas seguintes etapas: 1) Identificação de diretrizes propostas em três instrumentos (Sousa, Turrini, \& Poveda, 2015; Castro et al., 2007; Luz et al., 2003); 2) Compilação de diretrizes propostas na literatura nacional e internacional sobre a temática; 3) Levantamento de diretrizes a partir do discurso dos usuários do SUS que avaliaram três materiais educativos do Governo Federal (uma filipeta sobre câncer do intestino, uma cartilha sobre hipertensão e o capítulo dois do Guia Alimentar para Brasileiros, última edição) (Vasconcelos, Sampaio, \& Vergara, 2018).

Para a seleção dos juízes, realizou-se busca na plataforma Lattes do Conselho Nacional de Desenvolvimento Científico e Tecnológico (CNPq) utilizando os seguintes critérios de inclusão: possuir titulação de doutor e/ou mestre, atuar como docente em instituição de ensino superior e ter trabalhos publicados na área de interesse do estudo (letramento em saúde e/ou validação de instrumentos).

O grupo de juízes ficou composto por 14 especialistas, dos quais 01 era pedagogo, 03 nutricionistas e 10 enfermeiros. Quanto à qualificação profissional, 13 eram doutores e 1 mestre. Não há consenso na literatura quanto ao número de juízes, entretanto, o referencial teórico adotado aponta que no processo de validação de instrumentos é sugerido que se considere o número mínimo de seis avaliadores e o máximo de 20 (Pasquali, 2010).

O contato com os avaliadores foi realizado por meio de correio eletrônico, através de uma carta-convite, explicando sobre o objetivo do estudo e como se daria o processo de validação do AMEELS-BR. Foi enviado, anexo a carta, o Termo de Consentimento Livre e Esclarecido (TCLE) que deveria ser devolvido assinado, nos casos de aceite para participar da pesquisa. Após o recebimento do termo assinado pelo avaliador, foi enviada uma cópia do AMEELS-BR e a planilha de avaliação dos 53 itens do instrumento. 
Optou-se por realizar a validação do AMEELS-BR por meio da validação de conteúdo, que se refere ao grau em que o conteúdo do instrumento reflete adequadamente o construto que está sendo medido (Polit, 2015). A validade de conteúdo permite em um primeiro momento investigar cada item do instrumento de forma individual e em seguida o instrumento como um todo (Alexandre \& Coluci, 2011). Assim, foi pedido que os 14 juízes de forma individual verificassem se os itens do instrumento em questão eram compreensíveis (Pasquali, 2010).

Como não há teste estatístico para avaliar especificamente a validade do conteúdo, geralmente os pesquisadores usam uma abordagem qualitativa, através da avaliação de um comitê de especialistas, e, em seguida, uma abordagem quantitativa, descritiva, usando o Índice de Validade do Conteúdo (IVC) (Coluci, Alexandre, \& Milani, 2015).

Dessa forma, a avaliação dos itens do instrumento foi realizada por meio de classificação item a item pela escala Likert, um tipo de escala amplamente utilizada em questionários e pesquisas de opinião (Alexandre \& Coluci, 2011). Segundo Pasquali (2010), a escala Likert apresenta normalmente 3 ou mais pontos, em que o juiz deve dizer se concorda, discorda, ou está em dúvida se o que é afirmado no item é capaz de medir o que se propõe. Para esse estudo, utilizou-se uma escala de 05 pontos, sendo 1 equivalente a "discordo totalmente", 2 a "discordo em parte", 3 "não concordo, nem discordo", 4 "concordo parcialmente" e 5 "concordo totalmente".

Cada juiz avaliou o instrumento item a item indicando seu grau de concordância com a clareza sobre o que cada item se propunha a medir. Para avaliar a pertinência de cada item, utilizou-se como critério uma concordância entre os juízes de no mínimo 0,78 (Coluci, Alexandre, \& Milani, 2015). Além disso, cada item continha também um espaço livre onde o avaliador poderia acrescentar sugestões sobre alterações, dúvidas ou sugestões de melhora para aquele item.

A concordância entre os juízes foi verificada de forma quantitativa por meio do Índice de Validade de Conteúdo (IVC). Esse índice mede a porcentagem de juízes que estão em concordância sobre determinados aspectos do instrumento e de cada um de seus itens (Alexandre \& Coluci, 2011).

Assim, para calcular o índice foi feita a somatória das respostas "4" e "5" em cada item do questionário e dividido esta soma pelo número total de respostas (Grant \& Davis, 1997).

$$
\text { IVC }=\frac{\text { Número de respostas }{ }^{~} 4^{x} \text { ou " }{ }^{5} 5^{x}}{\text { Número total de respostas }}
$$

Os itens com pontuação "1" ou "2" podem ser revisados ou eliminados e taxa de concordância aceitável entre os juízes para avaliação dos itens individualmente deveria ser superior a 0,78 , enquanto para a verificação da validade do novo instrumento de uma forma geral deve haver uma concordância mínima de 0,80 (Coluci, Alexandre, \& Milani, 2015). Os itens que obtiveram IVC inferior a 0,78 foram reformulados, nenhum excluído. As respostas obtidas na coluna reservada a comentários e sugestões foram utilizadas para a reformulação desses itens.

Para o cálculo do IVC de cada item foram desconsideradas as avaliações em que o comentário do juiz demonstrou que o critério de avaliação (avaliar o item quanto à sua clareza) não foi atendido.

Além disso, procedeu-se o cálculo do IVC geral do instrumento por meio da média dos valores dos itens calculados separadamente, isso é, da soma de todos os IVC calculados separadamente dividido pelo número de itens considerados na avaliação (Polit \& Beck, 2006).

O projeto atende à Resolução (466/12-Brasil, 2012) e foi iniciado somente após aprovação pelo Comitê de Ética em Pesquisa com Seres Humanos da Universidade Estadual do Ceará (UECE). O número do parecer do CEPE é no 3.363.863. Todos os participantes, que aceitaram participar do estudo, assinaram o Termo de Consentimento Livre e Esclarecido (TCLE). 


\section{Resultados}

Participaram do estudo 14 juízes, com predominância do sexo feminino $(n=13)$, sendo 01 do sexo masculino. Quanto à formação, 01 era pedagogo, 03 nutricionistas e 10 enfermeiros. Além disso, 13 apresentavam título de doutor e 01 de mestre. Todos eram docentes em instituições de ensino superior e possuíam experiência prévia em estudos de validação e/ou letramento em saúde.

Em se tratando do processo de julgamento dos 53 itens que compõem o instrumento, com relação ao nível de compreensão, 39 itens obtiveram concordância dentro do nível estabelecido (IVC >0,78), enquanto 14 não obtiveram o nível de concordância necessário, com valores de IVC entre 0,54 e 0,77 (Quadro 1).

Quadro 1. Índice de Validade de Conteúdo (IVC) dos itens do AMEELS - BR. Fortaleza, Ceará, 2021.

\begin{tabular}{|c|c|}
\hline Itens do AMEELS-BR & IVC \\
\hline 1. O propósito está evidente & 92,31 \\
\hline 2. O conteúdo trata de comportamentos & 85,71 \\
\hline 3. O conteúdo está focado no propósito & 100 \\
\hline 4. O conteúdo destaca pontos principais & 92,31 \\
\hline 5. Usa poucas ideias principais & 83,33 \\
\hline 6. O conteúdo é cientificamente comprovado & 84,61 \\
\hline 7. Informa nome do autor e ano de publicação & 92,86 \\
\hline 8. Evita apelos e recomendações tendentes a criar demandas que, eventualmente, não possam ser atendidas & 69,23 \\
\hline 9. Não usa citação de pesquisa, estatística, instituição e especialista na área & 81,82 \\
\hline 10. Excesso de definições ou informações desnecessárias & 84,62 \\
\hline 11. A linguagem é neutra & 75 \\
\hline 12. A sequência de informações é consistente, fácil predizer o fluxo & 92,31 \\
\hline 13. Os termos técnicos, quando usados, são definidos & 85,71 \\
\hline 14. Os títulos e subtítulos são claros e informativos & 92,86 \\
\hline 15. As ideias e os conceitos abstratos, quando utilizados, devem ser acompanhados de exemplos. & 90,91 \\
\hline 16. Nível de leitura (FREport) Obs: Considerar “a, o, com, de, se...” palavras. Obs: Analisar o Anexo 1. & 57,14 \\
\hline 17. Usa escrita no estilo conversação e voz ativa & 78,57 \\
\hline 18. Usa vocabulário com palavras comuns no texto & 84,62 \\
\hline 19. O contexto vem antes de novas informações & 100 \\
\hline 20. O aprendizado é facilitado por tópicos (informa o que estar por vir) & 92,31 \\
\hline 21. Evita linguagem paternalista ou de julgamento & 75 \\
\hline 22. Usa parágrafos curtos & 85,71 \\
\hline 23. Usa poucas ideias por sentença & 78,57 \\
\hline 24. Usa palavras curtas & 76,92 \\
\hline 25. Coloca informações mais importantes no início & 78,57 \\
\hline 26. Diz o que deve fazer e evita dizer o que não deve & 81,82 \\
\hline 27. A linguagem deve ser compreensível, explicativa e adequada à população & 90 \\
\hline 28. Não deve existir ideia preconceituosa & 81,82 \\
\hline 29. Os conceitos e ideias devem ser claros e concisos; & 91,67 \\
\hline 30. Não são usadas palavras ou sentenças com sentido dúbio & 90,91 \\
\hline 31. Deve evitar abreviaturas. & 69,23 \\
\hline 32. O propósito da capa referente ao texto está claro & 78,57 \\
\hline 33. O propósito das demais ilustrações referentes ao texto está claro & 78,57 \\
\hline
\end{tabular}


Research, Society and Development, v. 10, n. 12, e68101220104, 2021

(CC BY 4.0) | ISSN 2525-3409 | DOI: http://dx.doi.org/10.33448/rsd-v10i12.20104

\begin{tabular}{|l|l|}
\hline 34. Tipos de ilustrações & 53,85 \\
\hline 35. As figuras/ilustrações são relevantes & 92,86 \\
\hline 36. As listas, tabelas, etc. são autoexplicativas & 92,31 \\
\hline 37. As ilustrações têm legenda & 92,31 \\
\hline 38. Cada ilustração corresponde a 01 mensagem & 85,71 \\
\hline 39. O número de ilustrações não é excessivo & 92,86 \\
\hline 40. As ilustrações são de boa qualidade (alta resolução) & 92,86 \\
\hline 41. As ilustrações quando corresponde à sequência, estão numeradas & 78,57 \\
\hline 42. Evita ilustrações que suscitem polêmica, quando não é o objetivo & 81,82 \\
\hline 43. Evita imagens "fortes", de apelo negativo & 75 \\
\hline 44. Características de layout & 61,54 \\
\hline 45. Tamanho e tipo de letra & 75 \\
\hline 46. São utilizados subtítulos & 78,57 \\
\hline 47. Utiliza a interação & 78,57 \\
\hline 48. As orientações são específicas e dão exemplos & 84,62 \\
\hline 49. Motivação e autoeficácia (subdivisão em pequenas partes) & 58,33 \\
\hline 50. Mensagens ou informações são mediatizadas pelas emoções e reações humanas e/ou mediatizadas por \\
porta-voz famoso & 58,33 \\
\hline 51. Uso apropriado de humor nas mensagens & 78,57 \\
\hline 52. É semelhante a sua lógica, linguagem e experiência & 61,54 \\
\hline 53. Imagem cultural e exemplos & 58,33 \\
\hline
\end{tabular}

Fonte: Autores (2021).

Os itens que não atingiram o IVC necessário foram reformulados (Quadro 2). Vale ressaltar que durante o processo de reformulação dos itens foram levados em conta os comentários e sugestões dos avaliadores.

As sugestões e comentários dos especialistas envolveram questões como reformulação de redação, redução de texto, desmembramento de itens, agrupamento de itens, substituição de palavras, acréscimo de informações e semelhança entre itens. Ainda no que se refere aos comentários dos juízes, uma vez que avaliada a sua pertinência, as sugestões dos especialistas foram acatadas. $\mathrm{O}$ instrumento final ficou com 55 itens, pois 02 itens foram subdivididos acatando recomendação dos juízes. 
Quadro 2. Itens do AMEELS- BR após reformulação segundo sugestões e comentários dos avaliadores. Fortaleza,

Ceará, 2021.

\begin{tabular}{|c|c|}
\hline Itens iniciais & Itens reformulados \\
\hline $\begin{array}{l}\text { 8. Evita apelos e recomendações tendentes a criar } \\
\text { demandas que, eventualmente, não possam ser } \\
\text { atendidas. }\end{array}$ & $\begin{array}{l}\text { 8. Apresenta recomendações que possam ser atendidas pelo serviço de } \\
\text { saúde. }\end{array}$ \\
\hline 11. A linguagem é neutra. & $\begin{array}{l}\text { 11. A linguagem é neutra (sem adjetivos comparativos, sem ser } \\
\text { promocional e sem apelos que não são verdadeiros). }\end{array}$ \\
\hline $\begin{array}{l}\text { 16. Nível de leitura (FREport) Obs: Considerar "a, o, } \\
\text { com, de, se..." palavras. Obs: Analisar o Anexo } 1 .\end{array}$ & $\begin{array}{l}\text { 16. A leitura é fácil para um indivíduo que cursou até o ensino } \\
\text { fundamental. }\end{array}$ \\
\hline \multirow[t]{2}{*}{ 21. Evita linguagem paternalista ou de julgamento. } & $\begin{array}{l}\text { 21. Usa linguagem não autoritária que promove autonomia e liberdade } \\
\text { do indivíduo }\end{array}$ \\
\hline & 21. Utiliza linguagem que evita julgar o indivíduo \\
\hline 24. Usa palavras curtas. & 24. Usa palavras curtas ( 01 ou 02 sílabas) predominantemente. \\
\hline 31. Deve evitar abreviaturas. & 31. As abreviaturas são evitadas. \\
\hline 34. Tipos de ilustrações. & $\begin{array}{l}\text { 34. As ilustrações são simples, apropriadas e familiares ao público- } \\
\text { alvo. }\end{array}$ \\
\hline 43. Evita imagens "fortes", de apelo negativo. & 43. As imagens utilizadas evitam apelo negativo. \\
\hline 44. Características de layout. & $\begin{array}{l}\text { 44. O layout é adequado (espaço em branco adequado, uso da cor não é } \\
\text { distração, as ilustrações estão ao lado do texto relacionado, sinaliza os } \\
\text { conteúdos importantes por seta ou por outro dispositivo visual). }\end{array}$ \\
\hline \multirow{2}{*}{ 45. Tamanho e tipo de letra } & 45.0 tamanho da letra é adequado (mínimo 12) \\
\hline & 45. O tipo de letra é adequado (serif maiúsculas e minúsculas) \\
\hline $\begin{array}{l}\text { 49. Motivação e autoeficácia } \\
\text { pequenas partes) }\end{array}$ & 49. O conteúdo é subdividido em pequenas partes \\
\hline $\begin{array}{l}\text { 50. Mensagens ou informações são mediatizadas } \\
\text { pelas emoções e reações humanas e/ou mediatizadas } \\
\text { por porta-voz famoso }\end{array}$ & 50. As mensagens são comunicadas por uma pessoa famosa \\
\hline $\begin{array}{l}\text { 52. É semelhante a sua lógica, linguagem e } \\
\text { experiência }\end{array}$ & 52. A mensagem é culturalmente apropriada ao público-alvo \\
\hline 53. Imagem cultural e exemplos & $\begin{array}{l}\text { 53. Usa imagens e exemplos que expressam a cultura local } \\
\text { positivamente }\end{array}$ \\
\hline
\end{tabular}

Fonte: Autores (2021).

Quanto à avaliação geral do instrumento, resultou em IVC $=0,81$, quando realizado por meio da soma de todos os IVC calculados separadamente dividido pelo número de itens considerado na avaliação.

O instrumento final ficou composto por 56 itens, divididos em seis domínios (conteúdo; linguagem; ilustrações; layout, tipografia e apresentação; estímulo/motivação do aprendizado; adequação cultural) (Quadro 3). Cada item deve ser classificado em 0 (não adequado), 1 (adequado) ou 2 (ótimo). A interpretação da pontuação do instrumento envolve os conceitos ótimo (70 a 100\%), adequado (40 a 69\%) ou não-aceitável (0 a 39\%), cujo cálculo pode ser visualizado nas orientações após o Quadro 3. 
Quadro 3 - Instrumento de Avaliação de Materiais Educativos Impressos com foco no Letramento em Saúde para o Brasil (AMEELS-BR). Fortaleza, Ceará, 2021.

\begin{tabular}{|c|c|c|c|}
\hline A. Conteúdo & 0 & 1 & 2 \\
\hline \multicolumn{4}{|l|}{ 1. O propósito está evidente } \\
\hline \multicolumn{4}{|l|}{ 2. O conteúdo trata de comportamentos } \\
\hline \multicolumn{4}{|l|}{ 3. O conteúdo está focado no propósito } \\
\hline \multicolumn{4}{|l|}{ 4. O conteúdo destaca pontos principais } \\
\hline \multicolumn{4}{|l|}{ 5. Usa poucas ideias principais } \\
\hline \multicolumn{4}{|l|}{ 6. O conteúdo é cientificamente comprovado } \\
\hline \multicolumn{4}{|l|}{ 7. Informa nome do autor e ano de publicação } \\
\hline \multicolumn{4}{|l|}{ 8. Apresenta recomendações que possam ser atendidas pelo serviço de saúde } \\
\hline \multicolumn{4}{|l|}{ 9. Não usa citação de pesquisa, estatística, instituição e especialista na área } \\
\hline \multicolumn{4}{|c|}{ 10. Excesso de definições ou informações desnecessárias } \\
\hline \multicolumn{4}{|c|}{$\begin{array}{l}\text { 11.A linguagem é neutra (sem adjetivos comparativos, sem ser promocional e sem apelos que não são } \\
\text { verdadeiros). }\end{array}$} \\
\hline \multicolumn{4}{|l|}{ 12. A sequência de informações é consistente, fácil predizer o fluxo } \\
\hline \multicolumn{4}{|l|}{ 13. Os termos técnicos, quando usados, são definidos } \\
\hline \multicolumn{4}{|l|}{ 14. Os títulos e subtítulos são claros e informativos } \\
\hline \multicolumn{4}{|c|}{ 15. As ideias e os conceitos abstratos, quando utilizados, devem ser acompanhados de exemplos. } \\
\hline \multirow{2}{*}{\multicolumn{4}{|c|}{$\begin{array}{l}\text { B. Linguagem } \\
\text { 16. A leitura é fácil para um indivíduo que cursou até o ensino fundamental }\end{array}$}} \\
\hline & & & \\
\hline \multicolumn{4}{|l|}{ 17. Usa escrita no estilo conversação e voz ativa } \\
\hline \multicolumn{4}{|l|}{ 18. Usa vocabulário com palavras comuns no texto } \\
\hline \multicolumn{4}{|l|}{ 19. O contexto vem antes de novas informações } \\
\hline \multicolumn{4}{|c|}{ 20. O aprendizado é facilitado por tópicos (informa o que estar por vir) } \\
\hline \multicolumn{4}{|c|}{ 21. Usa linguagem não autoritária, que promove autonomia e liberdade do indivíduo } \\
\hline \multicolumn{4}{|c|}{ 22. Utiliza linguagem que evita julgar o indivíduo } \\
\hline \multicolumn{4}{|l|}{ 23. Usa parágrafos curtos } \\
\hline \multicolumn{4}{|l|}{ 24. Usa poucas ideias por sentença } \\
\hline \multicolumn{4}{|l|}{ 25. Usa palavras curtas (01 ou 02 sílabas) predominantemente } \\
\hline 26. Coloca informações mais importantes no início & & & \\
\hline 27. Diz o que deve fazer e evita dizer o que não deve & & & \\
\hline 28. A linguagem deve ser compreensível, explicativa e adequada à po & & & \\
\hline 29. Não deve existir ideia preconceituosa & & & \\
\hline 30. Os conceitos e ideias devem ser claros e concisos; & & & \\
\hline 31. Não são usadas palavras ou sentenças com sentido dúbio & & & \\
\hline 32. As abreviaturas são evitadas & & & \\
\hline C. $\quad$ Ilustrações & & & \\
\hline 33. O propósito da capa referente ao texto está claro & & & \\
\hline 34. O propósito das demais ilustrações referentes ao texto está claro & & & \\
\hline 35. As ilustrações são simples, apropriadas e familiares ao público-al & & & \\
\hline 36. As figuras/ilustrações são relevantes & & & \\
\hline 37. As listas, tabelas, etc. são autoexplicativas & & & \\
\hline 38. As ilustrações têm legenda & & & \\
\hline 39. Cada ilustração corresponde a 01 mensagem & & & \\
\hline 40 O número de ilustrações não é excessivo & & & \\
\hline 41. As ilustrações são de boa qualidade (alta resolução) & & & \\
\hline 42. As ilustrações quando corresponde à sequência, estão numeradas & & & \\
\hline
\end{tabular}




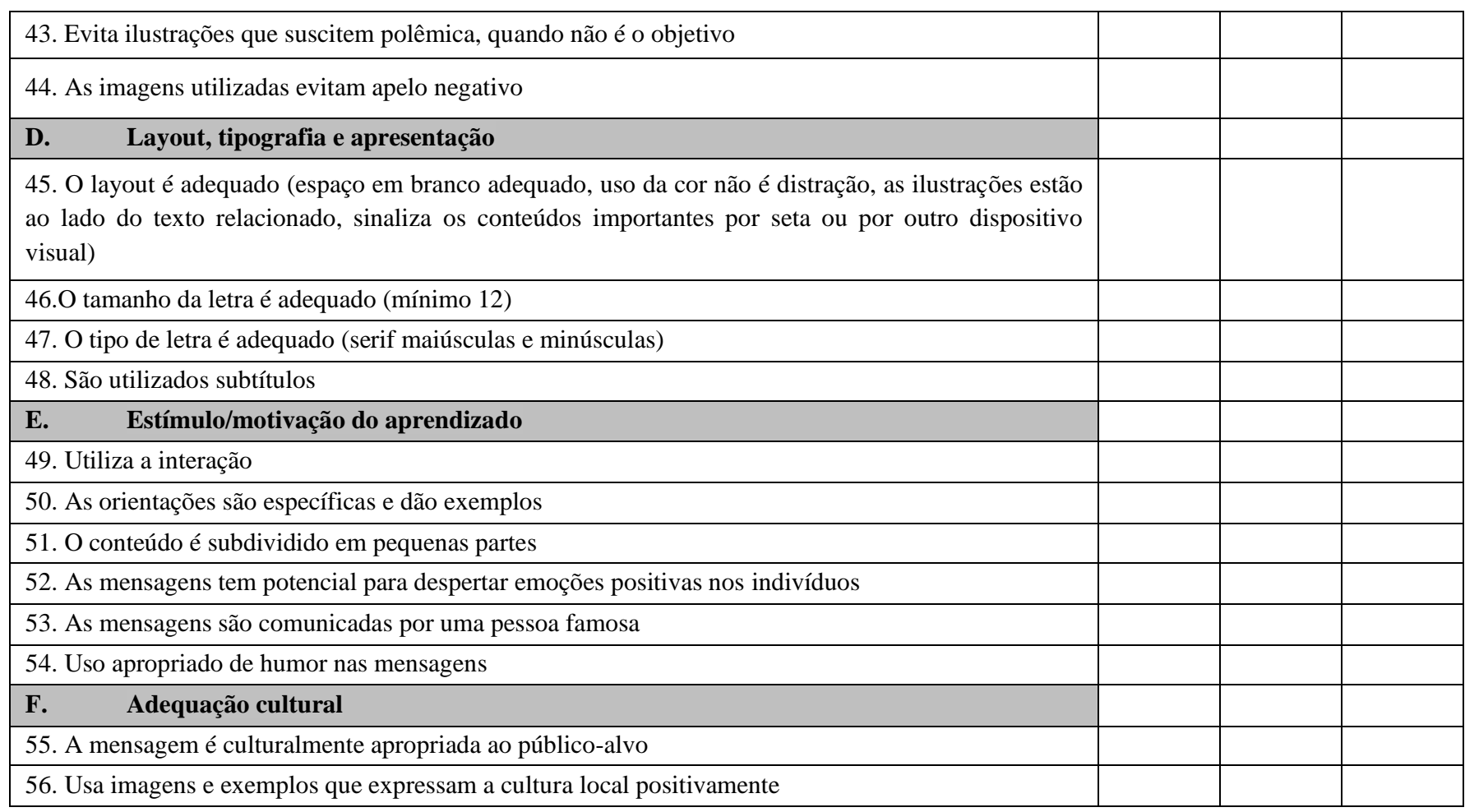

Fonte: Autores (2021).

Pontuação escore: 0 ponto para não adequado; 1 ponto para adequado; 2 pontos para ótimo; NA se o item não pode ser avaliado

$\mathrm{S}=$ Pontuação total AMEELS-BR (soma de todos fatores)

$\mathrm{M}=$ Pontuação máxima total $=112$

$\mathrm{N}=$ Número de respostas $\mathrm{N} / \mathrm{As}$ acima $=\ldots \mathrm{X} 2=$

$\mathrm{T}=$ Pontuação máxima total ajustada $=(\mathrm{M}-\mathrm{N})$

Percentual de pontuação $=\mathrm{S} / \mathrm{T}$

Interpretação da pontuação adequada

$\square$ Ótimo (70 a 100\%) $\square$ Adequado (40 a 69\%) $\square$ Não-aceitável (0 a 39\%)

\section{Discussão}

Visto que a literatura recomenda uma taxa de concordância não inferior a 0,78 na avaliação individual dos itens no caso da participação de seis ou mais juízes (Polit \& Beck, 2006), orientando que os itens com IVC inferior a esse valor devem ser revisados ou eliminados (Coluci, Alexandre, \& Milani, 2015), considera-se que o AMEELS-BR atingiu níveis satisfatórios de validade de conteúdo para 39 de seus itens.

Ainda, o instrumento apresentou a concordância mínima para a verificação da validade de um novo instrumento, que de uma forma geral não deve ser inferior a 0,8 (Polit \& Beck, 2006), sendo considerado válido quanto ao conteúdo, pois demonstra que realmente mensurou o que se propôs (Pasquali, 2010). Nesse contexto, o AMEELS-BR atingiu um nível satisfatório de validade quando avaliado de forma geral, demonstrando boa concordância entre juízes em relação à compreensão do instrumento.

Pode-se então considerar que o AMEELS, no que se refere ao seu conteúdo, é um instrumento válido para auxiliar os gestores e profissionais de saúde na elaboração e avaliação de materiais educativos impressos. É importante ressaltar que o AMEELS-BR, em sua construção, contemplou diretrizes propostas para avaliação de material educativo no Brasil com foco no 
letramento em saúde, constituindo-se assim de uma ferramenta útil para a melhoria da comunicação com os usuários dos serviços de saúde no Brasil, posto que considera o nível de letramento em saúde da população.

Antes de serem considerados aptos para uso, os instrumentos devem oferecer dados precisos, válidos e interpretáveis para a avaliação de saúde da população (Souza, Alexandre, \& Guirardello, 2017). Visto que a legitimidade e a credibilidade dos resultados de uma pesquisa têm como aspecto fundamental o reconhecimento da qualidade dos instrumentos, é reforçada a importância do processo de validação (Medeiros et al., 2018). Assim, a validação do AMEELS-BR é uma etapa importante para sua consolidação como instrumento interessante para a prática dos gestores, profissionais de saúde, elaboradores de materiais educativos, sendo considerada ferramenta inovadora a ser empregada para avaliar e subsidiar a elaboração dos MEI.

No que diz respeito aos instrumentos para avaliação de MEI, a literatura pesquisada nesse campo menciona instrumentos para avaliação em estudos estrangeiros, evidenciando a inexistência de uma ferramenta validada no Brasil que contemple os pressupostos do LS (Passamai et al., 2012; Vasconcelos, Sampaio, \& Vergara, 2018). Esse estudo pode ser considerado então como o primeiro no país a propor a validação de um instrumento que avalie os MEI sob os pressupostos do letramento em saúde para o Brasil.

Conforme mencionado, já existem instrumentos para avaliação de materiais educativos em outras línguas, como o SAM (Doak \& Doak, 1996), que vem sendo utilizado em pesquisas brasileiras a partir de sua tradução e adaptação em língua portuguesa (Sousa, Turrini, \& Poveda, 2015), mas que não possui ainda validação para o Brasil. Além do SAM, Vasconcelos, Sampaio, \& Vergara (2018) identificaram e revisaram em seu estudo instrumentos com proposta semelhante, destacando as ferramentas Presentation and Design - Document Perspective Baker Able Leaflet Design (BALD), Medication Information Design Assessment Scale (MIDAS), Readability and Presentation - Document Perspective Readability Assessment Instrument (RAIN), Quality - Document Perspective DISCERN, Ensuring Quality Information for Patients Scale (EQIP), Comprehensibility and presentation - Document and user perspectives consumer evaluation form (CEF), Comprehensibility, utility, design quality - Document and user perspective consumer information rating form (CIRF) e Satisfaction - User perspective satisfaction with information about Medicines Scale (SIMS).

Em âmbito nacional foi encontrado o Evaluation of Printed Education Materials (EVALPEM), que se trata de um instrumento adaptado do estrangeiro The Bernier Instructional Design Scale (BIDS) para avaliação de materiais educativos validado junto a profissionais de saúde para uso no Brasil (Castro et al., 2007). Outro instrumento utilizado para elaboração e o aperfeiçoamento de materiais educativos em saúde é o Questionário Luz et al. (2003), entretanto, diferentemente dos anteriormente citados, permite apenas uma avaliação qualitativa do impresso, visto que não atribui uma pontuação específica para classificação do material educativo ao final (Vasconcelos, Sampaio, \& Vergaa., 2018).

Assim, observa-se na literatura científica pesquisada ausência de um instrumento brasileiro validado para avaliação de MEI que contemple os pressupostos do letramento em saúde. Como agravante, apesar de os materiais educativos impressos serem comumente utilizados na promoção da saúde, tanto em instituições públicas como em privadas, a literatura tem relatado baixa qualidade desses materiais, evidenciando a necessidade da elaboração de instrumentos que os avaliem (Castro et al., 2007).

O AMEELS-BR se diferencia das demais ferramentas apresentadas por considerar os pressupostos do letramento em saúde, visto que foi elaborado com base em diretrizes propostas para avaliação de material educativo no Brasil nesse foco, permitindo assim a avaliação e elaboração desses materiais em acordo as características do público-alvo, tornando mais eficiente a comunicação com os leitores.

Compreendendo que instrumentos com diversas finalidades integram a prática clínica e a pesquisa em diferentes áreas do conhecimento, a avaliação de sua qualidade é fundamental para a seleção de instrumentos que forneçam medidas válidas e confiáveis (Schmitter-Edgecombe, Parsey, \& Lamb, 2014). Entretanto, é necessário que esses instrumentos apresentem 
qualidades psicométricas que garantam a sua confiabilidade, como a validade e reprodutibilidade (Echevarría-Guanilo, Gonçalves, \& Romanoski, 2017).

A validade de um instrumento diz respeito à capacidade do mesmo de medir com precisão o fenômeno a ser estudado (Pittman \& Bakas, 2010). Os psicometristas mencionam três métodos principais para obtenção da validade de uma medida, que são a validade de construto, a validade de critério e a validade de conteúdo (Pasquali, 2009).

A validade de construto é o grau em que um grupo de variáveis realmente representa o construto a ser medido (Hair et al., 2009). A validade de critério é a relação entre a pontuação de um determinado instrumento e outra externa, é o grau em que os resultados do questionário são um reflexo adequado de um "padrão ouro" (Rivière et al., 2018). Já a validade do conteúdo refere-se ao grau em que o conteúdo do instrumento reflete adequadamente o construto que está sendo medido, ou seja, avalia quanto uma amostra de itens representa em um universo ou domínio de conteúdo definido (Polit, 2015).

O AMEELS-BR já foi avaliado quanto a sua reprodutibilidade (Nascimento et al., 2018), obtendo bons resultados e se mostrando reprodutível na população estudada. Assim, após passar pelo processo de validação de conteúdo proposto neste estudo e ter sido, de forma geral, julgado adequado pelos especialistas, considera-se, que o instrumento conseguiu atender os propósitos para o qual foi elaborado.

A principal limitação deste estudo encontra-se no fato de se tratar de uma validação de conteúdo, que apesar de ser uma etapa fundamental no processo de desenvolvimento e adaptação de instrumento de medidas, apresenta limitações por ser um processo subjetivo. Também são necessárias aplicação de outras medidas psicométricas adicionais (Alexandre \& Coluci, 2011). No entanto, pode-se considerar que este instrumento é válido quanto ao seu conteúdo para avaliar os MEI sob a luz dos pressupostos do letramento em saúde.

\section{Conclusão}

Por meio do estudo realizado foi possível concluir que o AMEELS-BR, de forma geral, foi considerado valido quanto ao seu conteúdo pelo painel de especialistas, apresentando índice de validade dentro dos parâmetros aceitáveis e podendo assim ser utilizado por gestores e profissionais de saúde para a avaliação e elaboração dos MEI.

Apesar de resultados favoráveis, é importante que o instrumento passe posteriormente pelos processos de validação de constructo e de critério, além da aplicação de outras medidas psicométricas adicionais. Além disso, sugere-se que estudos futuros avaliem a usabilidade e aplicabilidade do instrumento pelos gestores e profissionais de saúde.

\section{Referências}

Castro, M. S., Pilger, D., Fuchs, D. F., \& Cardoso Ferreira, M. B. (2007). Development and validity of a method for the evaluation of printed education material. Pharmacy Practice, 5(2), 89-94. https://doi.org/10.4321/s1886-36552007000200007

Coluci, M. Z. O., Alexandre, N. M. C., \& Milani, D. (2015). Construção de instrumentos de medida na área da saúde. Ciência \& Saúde Coletiva, 20(3), 925936. https://doi.org/10.1590/1413-81232015203.04332013

Alexandre, N. M. C., \& Coluci, M. Z. O. (2011). Validade de conteúdo nos processos de construção e adaptação de instrumentos de medidas. Revista Ciência \& Saúde Coletiva, 16(7), 3061-3068. https://doi.org/10.1590/S1413-81232011000800006

Doak, C. C., Doak, L. G., \& Root, J. H. (1996). Teaching patients with low literacy skills. AJN The American Journal of Nursing, 96(12), 16M. https://doi.org/10.1097/00000446- 199612000- 00022

Echevarría-Guanilo, M. E., Gonçalves, N., \& Romanoski, P. J. (2017). Propriedades psicométricas de instrumentos de medidas: bases conceituais e métodos de avaliação-Parte I. Texto \& Contexto-Enfermagem, 26 (4). https://doi.org/10.1590/0104-07072017001600017

Giguère, A., Zomahoun, H. T. V., Carmichael, P. H., Uwizeye, C. B., Légaré, F., Grimshaw, J. M., \& Massougbodji, J. (2020). Printed educational materials: effects on professional practice and healthcare outcomes. Cochrane Database of Systematic Reviews, 8, CD004398. https://doi.org/10.1002/14651858 .CD004398.pub4

Grant, J. S., \& Davis, L. L. (1997). Selection and use of content experts for instrument development. Research in Nursing \& Health, 20(3), 269-274. https://doi.org/10.1002/(sici)1098-240x(199706)20:3<269:aid-nur9>3.0.co;2-g 
Hair, J. F., Black, W. C., Babin, B. J., Anderson, R. E., \& Tatham, R. L. (2009). Análise Multivariada de Dados. Bookman editora.

Hoffmann, T., \& Worrall, L. (2004). Designing effective written health education materials: considerations for health professionals. Disability and Rehabilitation, 26(19), 1166-1173. https://doi.org/10.1080/09638280410001724816

Luz, Z. M. P. D., Pimenta, D. N., Rabello, A., \& Schall, V. (2003). Evaluation of informative materials on leishmaniasis distributed in Brazil: criteria and basis for the production and improvement of health education materials. Cadernos de Saúde Pública, 19(2), 561-569. https://doi.org/10.1590/S0102311 X2003000200023

Medeiros, R. K. S., Júnior, M. A. F., Pinto, D. P. D. S. R., Vitor, A. F., Santos, V. E. P., \& Barichello, E. (2015). Modelo de validação de conteúdo de Pasquali nas pesquisas em Enfermagem. Revista de Enfermagem Referência, 4(4), 127-135. https://doi.org/10.12707/RIV14009

Mialhe, F. L., \& da Costa Silva, C. M. (2008). Estratégias para a elaboração de impressos educativos em saúde bucal. Arquivos em Odontologia, 44(2), 81-87. https://periodicos.ufmg.br/index.php/arquivosemodontologia/article/view/3468

Nascimento, A. C., Carioca, A. A., Vasconcelos, C. M., Henriques, E. M., Sampaio, H. A., Freitas, L. A. (2018). Reprodutibilidade do Instrumento de Avaliação de Materiais Educativos Impressos no Brasil (AMEELS-BR). In Anais do XXIV Encontro de Iniciação à Pesquisa da UNIFOR. Fortaleza, Ceará. https://uol.unifor.br/oul/conteudosite/?cdConteudo=8824725

Nascimento, É. A., Tarcia, R. M. L., Magalhães, L. P., Soares, M. A. D. L., Suriano, M. L. F., \& Domenico, E. B. L. D. (2015). Folhetos educativos em saúde: estudo de recepção. Revista da Escola de Enfermagem da USP, 49 (3), 432-439. https://doi.org/10.1590/S0080-623420150000300011

Pasquali, L. (2009). Psychometrics. Revista da Escola de Enfermagem da USP, 43, 992-999. https://doi.org/10.1590/S0080-62342009000500002

Pasquali, L. (2010). Instrumentação Psicológica: Fundamentos e práticas, 165-198.

Passamai, M. D. P. B., Sampaio, H. A. D. C., Dias, A. M. I., \& Cabral, L. A. (2012). Letramento funcional em saúde: reflexões e conceitos sobre seu impacto na interação entre usuários, profissionais e sistema de saúde. Interface-Comunicação, Saúde, Educação, 16(41), 301-314. https://doi.org/10.1590/S141432832012005000027

Pereira, A. S., Shitsuka, D. M., Parreira, F. J., \& Shitsuka, R. (2018). Metodologia da pesquisa científica. http://repositorio.ufsm.br/handle/1/15824

Pittman, J., \& Bakas, T. (2010). Measurement and instrument design. Journal of Wound Ostomy \& Continence Nursing, 37(6), 603-607. https://doi.org/10.1097/won.0b013e3181f90a60

Polit, D. F. (2015). Assessing measurement in health: Beyond reliability and validity. International Journal of Nursing Studies, 52(11), 1746-1753. https://doi.org/10.1016/j.ijnurstu.2015.07.002

Polit, D. F., \& Beck, C. T. (2006). The content validity index: are you sure you know what's being reported? Critique and recommendations. Research in Nursing \& Health, 29(5), 489-497. https://doi.org/10.1002/nur.20147

Rivière, F., Widad, F. Z., Speyer, E., Erpelding, M. L., Escalon, H., \& Vuillemin, A. (2018). Reliability and validity of the French version of the global physical activity questionnaire. Journal of Sport and Health Science, 7(3), 339-345. https://doi.org/10.1016/j.jshs.2016.08.004

Rocha, P. C., \& Lemos, S. M. A. (2016). Aspectos conceituais e fatores associados ao Letramento Funcional em Saúde: revisão de literatura. Revista CEFAC, 18 (1), 214-225. https://doi.org/10.1590/1982-021620161819615

Santos, J. E. M. D., Brasil, V. V., Moraes, K. L., Cordeiro, J. A. B. L., Oliveira, G. F. D., Bernardes, C. D. P., Bueno, R. M. B., Boaventura, R. P., Gonçalves, F. A. F., Oliveira, L. M. A. C., Barbosa, M. A., \& Silva, A. M. T. C. (2017). Legibilidade de prospecto facilitador e letramento em saúde de indivíduos com marcapasso. Revista Brasileira de Enfermagem, 70(3), 633-639. https://doi.org/10.1590/0034-7167-2016-0336

Schmitter-Edgecombe, M., Parsey, C., \& Lamb, R. (2014). Development and psychometric properties of the instrumental activities of daily living: compensation scale. Archives of Clinical Neuropsychology, 29(8), 776-792. https://doi.org/10.1093/arclin/acu053

Sørensen, K., Van den Broucke, S., Fullam, J., Doyle, G., Pelikan, J., Slonska, Z., \& Brand, H. (2012). Health literacy and public health: a systematic review and integration of definitions and models. BMC Public Health, 12(1), 1-13. https://doi.org/10.1186/1471-2458-12-80

Sousa, C. S., Turrini, R. N. T., \& Poveda, V. B. (2015). Translation and adaptation of the instrument" suitability assessment of materials"(SAM) into Portuguese. Journal of Nursing UFPE on line, 9(5), 7854-7861. https://doi.org/10.5205/1981-8963-v9i5a10534p7854-7861-2015

Souza, A. C. D., Alexandre, N. M. C., \& Guirardello, E. D. B. (2017). Psychometric properties in instruments evaluation of reliability and validity. Epidemiologia e Serviços de Saúde, 26, 649-659. https://doi.org/10.5123/s1679-49742017000300022

Vasconcelos, C. M. C., Sampaio, H. A., Vergara, C. M. (2018). Materiais educativos para prevenção e controle de doenças crônicas: uma avaliação à luz dos pressupostos do letramento em saúde. CRV.

Vasconcelos, C. M. C. S. (2016). Materiais educativos para prevenção e controle de doenças crônicas: uma avaliação à luz dos pressupostos do letramento em saúde (Tese de doutorado). Universidade Estadual do Ceará, Fortaleza. CD-ROM. 